\title{
A novel index of infarct morphology predicts the presence of microvascular obstruction in patients with acute myocardial infarction
}

\author{
Lowie M Van Assche ${ }^{1 *}$, Han W Kim', Sebastiaan C Bekkers ${ }^{2}$, Brenda Hayes ${ }^{1}$, Michele Parker ${ }^{1}$, Raymond J Kim \\ From 15th Annual SCMR Scientific Sessions \\ Orlando, FL, USA. 2-5 February 2012
}

\section{Background}

Microvascular obstruction (MO) has been associated with poor LV remodeling and adverse prognosis. Infarct morphology is related to the presence of MO in that patients with $\mathrm{MO}$ generally have larger infarct size (IS) and greater mean infarct transmurality. However, neither index is highly predictive on an individual patient basis. In the current study, we investigated the utility of a novel index of infarct morphology, which reflects the circumferential extent of fully transmural infarction extending to the epicardial surface-the epicardial surface area (EpiSA) of infarction-to predict MO.

\section{Methods}

We studied 302 consecutive patients from 2 centers (Duke and Maastricht University) with first AMI. On contrast-enhanced-CMR, early (2-min post-contrast) and late MO (10-min post-contrast) were defined as hypoenhanced regions within hyperenhanced infarction. Infarct size, mean transmurality, and EpiSA were quantified by manual planimetry of the stack of short-axis views.

\section{Results}

Patients were $58 \pm 11$ years old ( $71 \%$ male). Prevalence of early and late MO was $64 \%$ and $55 \%$, respectively. For the population, IS, mean transmurality, and EpiSA were $14 \%$ of LV mass (IQR 7-25\%), 74\% of infarct sector (IQR $57-86 \%$ ) and $6 \%$ of total LV epicardial-surface-area (IQR 1-13\%), respectively. All 3 infarct characteristics were significantly larger in patients with MO (all $\mathrm{p}<0.0001)$. On ROC-curve analyses, EpiSA predicted

${ }^{1}$ Cardiology, Duke University, Durham, NC, USA

Full list of author information is available at the end of the article
MO more accurately (e.g. larger area-under-the-curve) then IS or mean transmurality (Figure 1). For the 3 infarct characteristics, Table 1 shows threshold, cut-off values for which MO was always absent or always present in the population. For instance, MO (early or late) was always absent when infarct size was $<1.4 \%$ of $\mathrm{LV}$ ( $5 \%$ of population), and always present when $>42 \%$ of LV (4\% of population). However, only a small portion of the population $(5 \%+4 \%=9 \%)$ had infarct size reaching these thresholds, showing that IS had limited discriminatory value on an individual patient basis. Similarly, infarct transmurality had limited discriminatory value. In contrast, EpiSA thresholds allowed ruling-in or ruling-out MO in a significantly larger percentage of the population ( $44 \%$ for both early and late; $\mathrm{p}<0.0001 \mathrm{com}$ pared with IS and transmurality). No patient had MO unless EpiSA was greater than zero. Multivariable analysis incorporating clinical, ECG, and CMR data demonstrated that EpiSA was the strongest, independent predictor of early and late MO ( $<<0.0001$ for both).

\section{Conclusions}

The epicardial surface area of infarction, a novel index of infarct morphology, is a stronger predictor of $\mathrm{MO}$ than infarct size or mean transmurality. MO does not occur unless infarction extends to the epicardial surface.

\section{Funding}

Funded in part by 5R01HL064726-07.

\section{Author details}

${ }^{1}$ Cardiology, Duke University, Durham, NC, USA. ${ }^{2}$ Cardiology, Maastricht University, Maastricht, Netherlands.

Published: 1 February 2012 
Figure 1
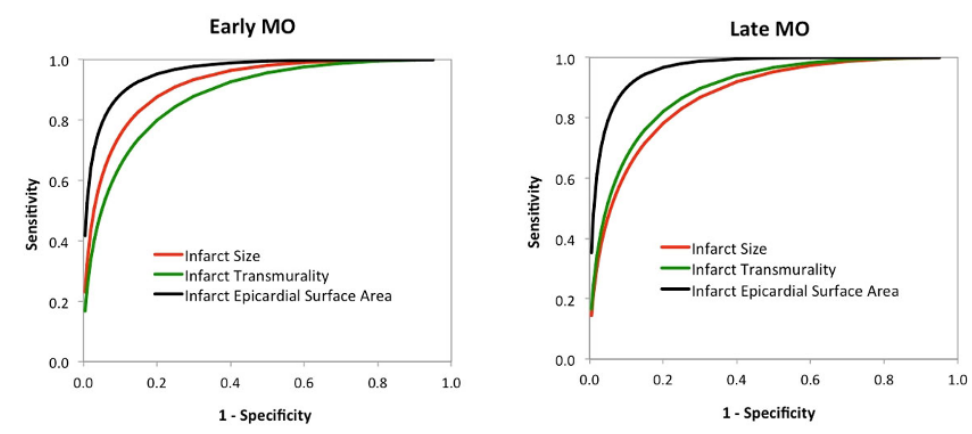

\begin{tabular}{c|c|c}
\hline Early MO & & Late MO \\
\hline Area Under the ROC Curve & Infarct Characteristic & Area Under the ROC Curve \\
\hline 0.92 & Size & 0.87 \\
0.88 & Transmurality & 0.89 \\
0.96 & Epicardial Surface Area & 0.96 \\
& & \\
\hline Pvalue & Comparisons & Pvalue \\
\hline 0.08 & Size vs Transmurality & 0.4 \\
0.006 & Size vs EpiSA & $<0.0001$ \\
\hline 0.0001 & Transmurality vs EpiSA & $<0.0001$ \\
\hline \multicolumn{2}{|c}{}
\end{tabular}

Figure 1

Table 1 Presence or absence of MO according to thresholds of infarct characteristics

\begin{tabular}{|c|c|c|c|}
\hline \multirow[b]{2}{*}{ Early MO } & \multicolumn{3}{|c|}{ Microvascular obstruction } \\
\hline & Always absent & Absent or present & Always present \\
\hline \multicolumn{4}{|l|}{ Infarct size } \\
\hline$\%$ LV threshold & $<1.4$ & $1.4-42$ & $>42$ \\
\hline$\%$ population represented & $5 \%$ & $92 \%$ & $4 \%$ \\
\hline \multicolumn{4}{|l|}{ Infarct transmurality } \\
\hline$\%$ LV threshold & $<35$ & $35-95$ & $>95$ \\
\hline$\%$ population represented & $4 \%$ & $89 \%$ & $7 \%$ \\
\hline \multicolumn{4}{|l|}{ Infarct epicardial surface area } \\
\hline$\%$ total LV surface area threshold & $<0.3$ & $0.3-13.6$ & $>13.7$ \\
\hline$\%$ population represented & $20 \% *$ & $56 \%$ & $24 \% *$ \\
\hline \multicolumn{4}{|l|}{ Late $\mathrm{MO}$} \\
\hline \multicolumn{4}{|l|}{ Infarct size } \\
\hline$\%$ LV threshold & $<1.4$ & $1.4-42$ & $>42$ \\
\hline$\%$ population represented & $5 \%$ & $92 \%$ & $4 \%$ \\
\hline \multicolumn{4}{|l|}{ Infarct transmurality } \\
\hline$\%$ LV threshold & $<35$ & $34-98$ & $>99$ \\
\hline$\%$ population represented & $4 \%$ & $95 \%$ & $1 \%$ \\
\hline \multicolumn{4}{|l|}{ Infarct epicardial surface area } \\
\hline$\%$ total LV surface area threshold & $<0.3$ & $0.3-13.6$ & $>13.7$ \\
\hline$\%$ population represented & $21 \% *$ & $56 \%$ & $23 \% *$ \\
\hline
\end{tabular}

*\% population represented is greater than corresponding population for infarct size and transmurality $(p<0.0001)$.

doi:10.1186/1532-429X-14-S1-P23

Cite this article as: Van Assche et al:: A novel index of infarct

morphology predicts the presence of microvascular obstruction in

patients with acute myocardial infarction. Journal of Cardiovascular

Magnetic Resonance 2012 14(Suppl 1):P23. 